ANNALES

POLONICI MATHEMATICI

$95.3(2009)$

\title{
Solutions for a class of hemivariational inequalities with $p(x)$-Laplacian
}

\author{
by Xia Zhang and YongQIANG Fu (Harbin)
}

\begin{abstract}
We study a class of hemivariational inequalities with $p(x)$-Laplacian. Applying nonsmooth critical point theory for locally Lipschitz functions, we obtain the existence of solutions on interior and exterior domains.
\end{abstract}

1. Introduction and main results. Since the paper by Kováčik and Rákosník [12] where the spaces $L^{p(x)}$ and $W^{1, p(x)}$ were thoroughly studied, variable exponent Sobolev spaces have been used extensively to model various phenomena. In [17] Růžička applied them in the study of electrorheological fluids. In recent years, the differential equations and variational problems with $p(x)$-growth conditions have been extensively investigated (see for example $[1,9,10,14]$ ).

Here we discuss a class of hemivariational inequalities with $p(x)$-Laplacian. Hemivariational inequalities arise in problems of mechanics and engineering, when one considers more realistic laws of nonmonotone and multivalued nature. For concrete applications, we refer to Naniewicz-Panagiotopoulos [15] and Panagiotopoulos [16]. In this paper, we study the following hemivariational inequality:

$$
\left\{\begin{array}{l}
u \in W_{0}^{1, p(x)}(\Omega), \\
\int_{\Omega}\left(|\nabla u|^{p(x)-2} \nabla u \nabla v+|u|^{p(x)-2} u v\right) d x \\
\quad+\int_{\Omega} F_{x}^{0}(x, u(x) ;-v(x)) d x \geq 0, \quad \forall v \in W_{0}^{1, p(x)}(\Omega),
\end{array}\right.
$$

where $\Omega \subset \mathbb{R}^{N}$ is a domain, $p$ is Lipschitz continuous on $\bar{\Omega}$ and satisfies $1<p_{-} \leq p(x) \leq p_{+}<N$.

2000 Mathematics Subject Classification: Primary 35J85.

Key words and phrases: hemivariational inequality, nonsmooth critical point theory, locally Lipschitz function, generalized gradient. 
Now we recall some basic properties of variable exponent spaces $L^{p(x)}(\Omega)$ and variable exponent Sobolev spaces $W^{1, p(x)}(\Omega)$, where $\Omega \subset \mathbb{R}^{N}$ is a domain. For a deeper treatment of these spaces, we refer to $[5,6,12]$.

Let $\mathbf{P}(\Omega)$ be the set of all Lebesgue measurable functions $p: \Omega \rightarrow[1, \infty)$ and for $p \in \mathbf{P}(\Omega)$ set

$$
|u|_{p(x)}=\inf \left\{\lambda>0: \int_{\Omega}|u / \lambda|^{p(x)} d x \leq 1\right\} .
$$

The variable exponent space $L^{p(x)}(\Omega)$ is the class of all functions $u$ such that $\int_{\Omega}|u(x)|^{p(x)} d x<\infty$; it is a Banach space equipped with the norm (1.2).

The variable exponent Sobolev space $W^{1, p(x)}(\Omega)$ is the class of all functions $u \in L^{p(x)}(\Omega)$ such that $|\nabla u| \in L^{p(x)}(\Omega)$; it can be equipped with the norm

$$
\|u\|_{1, p(x)}=|u|_{p(x)}+|\nabla u|_{p(x)} .
$$

We denote by $W_{0}^{1, p(x)}(\Omega)$ the subspace of $W^{1, p(x)}(\Omega)$ which is the closure of $C_{0}^{\infty}(\Omega)$ with respect to the norm (1.3); if $\Omega \subset \mathbb{R}^{N}$ is a bounded domain, then $\|u\|_{1, p(x)}$ and $|\nabla u|_{p(x)}$ are equivalent norms on $W_{0}^{1, p(x)}(\Omega)$.

For all $p \in \mathbf{P}(\Omega)$, we write

$$
p_{+}=\sup _{x \in \Omega} p(x), \quad p_{-}=\inf _{x \in \Omega} p(x),
$$

and denote by $p_{1} \ll p_{2}$ the fact that $\inf _{x \in \Omega}\left(p_{2}(x)-p_{1}(x)\right)>0$.

Throughout this paper, we assume that $F: \bar{\Omega} \times \mathbb{R} \rightarrow \mathbb{R}$ is a Carathéodory function which is locally Lipschitz in the second variable and $F(x, 0)=0$ for all $x \in \Omega . F_{x}^{0}(x, s ; z)$ is the generalized directional derivative of $F(x, \cdot)$ at $s \in \mathbb{R}$ in direction $z \in \mathbb{R}$.

In addition, we need various conditions on $F$ corresponding to the cases when $\Omega$ is an interior or exterior domain. Firstly, consider the case when $\Omega$ is an interior domain, i.e. $\Omega$ is bounded.

(H1) There exists $\alpha \in C(\bar{\Omega})$ with $p(x) \ll \alpha(x) \ll p^{*}(x)$ such that

$$
|\xi| \leq a_{0}+a_{1}|t|^{\alpha(x)-1}
$$

for all $(x, t) \in \Omega \times \mathbb{R}$ and $\xi \in \partial F(x, t)$, where $\partial F(x, t)$ is the generalized gradient of $F(x, \cdot)$ at $t \in \mathbb{R}$, and $a_{0}, a_{1}>0$.

(H2) There exists $p(x) \ll \mu$ such that $\mu F(x, t) \leq-F_{x}^{0}(x, t ;-t)$ for all $(x, t) \in \Omega \times \mathbb{R}$. Moreover, there exist an open set $\Omega_{0} \subset \Omega$ and $a_{2}, a_{3}>0$ such that $F(x, t) \geq a_{2}|t|^{\mu}-a_{3}$ for any $(x, t) \in \Omega_{0} \times \mathbb{R}$.

(H3) $\lim _{t \rightarrow 0} \max \{|\xi|: \xi \in \partial F(x, t)\} /|t|^{p(x)-1}=0$ uniformly for almost every $x \in \Omega$.

(H4) There exists $\beta \in \mathbf{P}(\Omega)$ with $1<\beta_{-} \leq \beta(x) \ll p(x)$ such that

$$
|\xi| \leq b_{0}+b_{1}|t|^{\beta(x)-1}
$$

for all $(x, t) \in \Omega \times \mathbb{R}$ and $\xi \in \partial F(x, t)$, where $b_{0}, b_{1}>0$. 
(H5) Let $\beta(x)$ be as in (H4). There exist $b_{2}>0,0<\delta<1$ and an open set $\Omega_{0} \subset \Omega$ such that $F(x, t) \geq b_{2}|t|^{\beta(x)}$ for all $(x, t) \in \Omega_{0} \times(0, \delta)$.

Under these conditions, we get the following results.

TheOREM 1.1. Let $\Omega \subset \mathbb{R}^{N}$ be a bounded domain. Under hypotheses (H1)-(H3), problem (1.1) has at least one non-trivial solution.

TheOREM 1.2. Let $\Omega \subset \mathbb{R}^{N}$ be a bounded domain. Under hypotheses (H4), (H5), problem (1.1) has at least one non-trivial solution.

We now consider the case when $\Omega$ is an exterior domain, i.e. the complement of a bounded domain.

(H6) $|\xi| \leq g(x)|t|^{\alpha(x)-1}$ for all $(x, t) \in \Omega \times \mathbb{R}$ and $\xi \in \partial F(x, t)$, where $\alpha \in \mathbf{P}(\Omega)$ with $p(x) \ll \alpha(x) \ll p^{*}(x), g(x) \geq 0$ and $g \in L^{\infty}(\Omega) \cap$ $L^{q_{1}(x)}(\Omega)$ with $q_{1}(x)=p^{*}(x) /\left(p^{*}(x)-\alpha(x)\right)$.

(H7) $|\xi| \leq h(x)|t|^{\beta(x)-1}$ for all $(x, t) \in \Omega \times \mathbb{R}$ and $\xi \in \partial F(x, t)$, where $\beta \in \mathbf{P}(\Omega)$ with $1<\beta_{-} \leq \beta(x) \ll p(x), h(x) \geq 0$ and $h \in L^{\infty}(\Omega) \cap$ $L^{q_{2}(x)}(\Omega)$ with $q_{2}(x)=p^{*}(x) /\left(p^{*}(x)-\beta(x)\right)$.

With these assumptions we have the following results.

TheOREM 1.3. Assume hypotheses (H2), (H6) hold and $\Omega \subset \mathbb{R}^{N}$ is an exterior domain. Then problem (1.1) has at least one non-trivial solution.

TheOREM 1.4. Assume hypotheses (H5), (H7) hold and $\Omega \subset \mathbb{R}^{N}$ is an exterior domain. Then problem (1.1) has at least one non-trivial solution.

2. Critical point theory for locally Lipschitz functions. In this paper, our approach is mainly based on variational methods for nondifferentiable functionals, namely, locally Lipschitz functionals. For a deeper treatment of this theory, we refer to $[2,3,4,13]$. Now we present some basic definitions and preliminary results.

Let $(X,\|\cdot\|)$ be a Banach space, $X^{*}$ its topological dual, and $\varphi: X \rightarrow \mathbb{R}$ a locally Lipschitz function. The generalized directional derivative of $\varphi$ at $u \in X$ in direction $v \in X$ is defined by

$$
\varphi^{0}(u ; v)=\limsup _{\substack{w \rightarrow u \\ t \rightarrow 0+}} \frac{\varphi(w+t v)-\varphi(w)}{t} .
$$

The generalized gradient of $\varphi$ at $u \in X$ is the set

$$
\partial \varphi(u)=\left\{w^{*} \in X^{*}:\left\langle w^{*}, v\right\rangle \leq \varphi^{0}(u ; v), \forall v \in X\right\},
$$

where $\langle\cdot, \cdot\rangle$ is the duality pairing between $X^{*}$ and $X$. A point $u \in X$ is a critical point of $\varphi$ if $0 \in \partial \varphi(u)$. If $u \in X$ is a critical point, the value $c=\varphi(u)$ is a critical value of $\varphi$. 
In the classical (smooth) theory, a basic analytical tool is a compactnesstype condition, known as the Palais-Smale condition. In the present nonsmooth setting this condition takes the following form: A locally Lipschitz function $\varphi: X \rightarrow \mathbb{R}$ satisfies the nonsmooth Palais-Smale (P.S.) condition if every sequence $\left\{u_{n}\right\} \subset X$ such that $\varphi\left(u_{n}\right)$ is bounded and

$$
\lambda\left(u_{n}\right)=\min \left\{\left\|w^{*}\right\|_{X^{*}}: w^{*} \in \partial \varphi\left(u_{n}\right)\right\} \rightarrow 0
$$

as $n \rightarrow \infty$, has a convergent subsequence in $X$.

Proposition 2.1. If $X$ is a reflexive Banach space, $\varphi: X \rightarrow \mathbb{R}$ is a locally Lipschitz function which satisfies the nonsmooth (P.S.) condition and for some $r>0$ and $x_{1}, x_{2}$ with $\left\|x_{1}-x_{2}\right\|_{X}>r$, we have

$$
\max \left\{\varphi\left(x_{1}\right), \varphi\left(x_{2}\right)\right\}<\inf \left\{\varphi(x):\left\|x-x_{1}\right\|_{X}=r\right\},
$$

then there exists a critical point $y_{0} \in X$ of $\varphi$ such that

$$
c=\varphi\left(y_{0}\right) \geq \inf \left\{\varphi(x):\left\|x-x_{1}\right\|_{X}=r\right\}
$$

and $c$ is defined by the following minimax formula:

$$
c=\inf _{\gamma \in \Gamma} \max _{t \in[0,1]} \varphi(\gamma(t)),
$$

where $\Gamma=\left\{\gamma \in C([0,1], X): \gamma(0)=x_{1}, \gamma(1)=x_{2}\right\}$.

Proposition 2.2. If $X$ is a reflexive Banach space, and $\varphi: X \rightarrow \mathbb{R}$ is a locally Lipschitz function which satisfies the nonsmooth (P.S.) condition and is bounded from below, then $c=\inf _{u \in X} \varphi(u)$ is a critical value of $\varphi$.

3. The case of interior domain. Throughout this section, we assume that $\Omega \subset \mathbb{R}^{N}$ is a bounded domain and denote by $c_{i}$ various positive constants. In order to discuss the problem (1.1), we need to define two functionals on $W_{0}^{1, p(x)}(\Omega)$ :

$$
\begin{aligned}
& \psi(u)=\int_{\Omega} F(x, u) d x \\
& \varphi(u)=J(u)-\psi(u)=\int_{\Omega} \frac{|\nabla u|^{p(x)}+|u|^{p(x)}}{p(x)} d x-\psi(u) .
\end{aligned}
$$

TheOREM 3.1. Under condition (H1) or (H4), $\psi$ is well defined and is a locally Lipschitz functional on $W_{0}^{1, p(x)}(\Omega)$.

Proof. Here we only consider the case where $F$ satisfies (H1).

(i) $\psi$ is well defined. For all $t_{1}, t_{2} \in \mathbb{R}$, by Lebourg's mean value theorem (see [4]), there exist $\theta \in(0,1)$ and $\xi_{\theta} \in \partial F\left(x, \theta t_{1}+(1-\theta) t_{2}\right)$ such that

$$
F\left(x, t_{1}\right)-F\left(x, t_{2}\right)=\xi_{\theta}\left(t_{1}-t_{2}\right)
$$


for all $x \in \Omega$. By condition (H1), we get

$$
\begin{aligned}
\left|F\left(x, t_{1}\right)-F\left(x, t_{2}\right)\right| & \leq\left(a_{0}+a_{1}\left|\theta t_{1}+(1-\theta) t_{2}\right|^{\alpha(x)-1}\right)\left|t_{1}-t_{2}\right| \\
& \leq\left(a_{0}+c_{1}\left|t_{1}\right|^{\alpha(x)-1}+c_{1}\left|t_{2}\right|^{\alpha(x)-1}\right)\left|t_{1}-t_{2}\right| .
\end{aligned}
$$

We also get

$$
|\psi(u)| \leq \int_{\Omega}|F(x, u)| d x \leq \int_{\Omega}\left(a_{0}+c_{1}|u|^{\alpha(x)-1}\right)|u| d x .
$$

By Theorem 1.1 in [8], we have $u \in L^{1}(\Omega)$ and $u \in L^{\alpha(x)}(\Omega)$ for all $u \in$ $W_{0}^{1, p(x)}(\Omega)$. Hence $|\psi(u)|<\infty$.

(ii) $\psi$ is locally Lipschitz on $W_{0}^{1, p(x)}(\Omega)$. Note that for all $u_{1}, u_{2} \in$ $W_{0}^{1, p(x)}(\Omega)$,

$$
\begin{aligned}
\left|\psi\left(u_{1}\right)-\psi\left(u_{2}\right)\right| & \leq \int_{\Omega}\left|F\left(x, u_{1}\right)-F\left(x, u_{2}\right)\right| d x \\
& \leq \int_{\Omega}\left(a_{0}+c_{1}\left|u_{1}\right|^{\alpha(x)-1}+c_{1}\left|u_{2}\right|^{\alpha(x)-1}\right)\left|u_{1}-u_{2}\right| d x \\
& \leq\left. c_{2}|1+| u_{1}\right|^{\alpha(x)-1}+\left.\left|u_{2}\right|^{\alpha(x)-1}\right|_{\alpha^{\prime}(x)} \cdot\left|u_{1}-u_{2}\right|_{\alpha(x)} \\
& \leq\left. c_{3}|1+| u_{1}\right|^{\alpha(x)-1}+\left.\left|u_{2}\right|^{\alpha(x)-1}\right|_{\alpha^{\prime}(x)} \cdot\left|\nabla u_{1}-\nabla u_{2}\right|_{p(x)} .
\end{aligned}
$$

Hence it is easy to get the result.

THEOREM 3.2. Under condition (H1) or $(\mathrm{H} 4)$, for all $u, v \in W_{0}^{1, p(x)}(\Omega)$ we have

$$
\psi^{0}(u ; v) \leq \int_{\Omega} F_{x}^{0}(x, u(x) ; v(x)) d x .
$$

Proof. We only consider the case where $F$ satisfies (H1).

(i) $\int_{\Omega} F_{x}^{0}(x, u(x) ; v(x)) d x<\infty$. In fact, $F(x, \cdot)$ is continuous for all $x \in \Omega$, thus

$$
\begin{aligned}
\limsup _{\substack{y \rightarrow u(x) \\
t \rightarrow 0+}} \frac{F(x, y+t v(x))-F(x, y)}{t} \\
=\limsup _{\substack{z \rightarrow 0+\\
t \rightarrow 0+}} \frac{F(x, z+u(x)+t v(x))-F(x, z+u(x))}{t} \\
=\limsup _{\substack{z_{n} \rightarrow 0 \\
t_{n} \rightarrow 0+}} \frac{F\left(x, z_{n}+u(x)+t_{n} v(x)\right)-F\left(x, z_{n}+u(x)\right)}{t_{n}},
\end{aligned}
$$

where $z_{n}, t_{n}$ are rational values. As $u(x), v(x)$ are measurable, we see that $F_{x}^{0}(x, u(x) ; v(x))$, being the "countable limsup" of measurable functionals of $x$, is also measurable. 
We know $F_{x}^{0}(x, u(x) ; v(x))=\max \{\xi \cdot v(x): \xi \in \partial F(x, u(x))\} \triangleq \xi_{x} \cdot v(x)$ for all $x \in \Omega$. By condition (H1), we get

$$
\left|F_{x}^{0}(x, u(x) ; v(x))\right|=\left|\xi_{x} \cdot v(x)\right| \leq a_{0}|v(x)|+a_{1}|v(x)| \cdot|u(x)|^{\alpha(x)-1},
$$

and so $F_{x}^{0}(x, u(x) ; v(x)) \in L^{1}(\Omega)$.

(ii) $\psi^{0}(u ; v) \leq \int_{\Omega} F_{x}^{0}(x, u(x) ; v(x)) d x$. By the definition of $\psi^{0}(u ; v)$, there exist $t_{n} \rightarrow 0+$ and $w_{n} \rightarrow u$ in $W_{0}^{1, p(x)}(\Omega)$ such that

$$
\varphi^{0}(u ; v)=\lim _{\substack{w_{n} \rightarrow u \\ t_{n} \rightarrow 0+}} \frac{\varphi\left(w_{n}+t_{n} v\right)-\varphi\left(w_{n}\right)}{t_{n}} .
$$

Passing to a subsequence, still denoted by $\left\{w_{n}\right\}$, we may assume that $w_{n}(x) \rightarrow u(x)$ a.e. in $\Omega$ as $n \rightarrow \infty$. Set

$$
\begin{aligned}
A_{n}(x) & =\frac{F\left(x, w_{n}(x)+t_{n} v(x)\right)-F\left(x, w_{n}(x)\right)}{t_{n}}, \\
B_{n}(x) & =\left(a_{0}+c_{1}\left|w_{n}(x)+t_{n} v(x)\right|^{\alpha(x)-1}+c_{1}\left|w_{n}(x)\right|^{\alpha(x)-1}\right)|v(x)|, \\
g_{n}(x) & =-A_{n}(x)+B_{n}(x) .
\end{aligned}
$$

It is easy to verify that $g_{n}(x) \geq 0$ for all $x \in \Omega$,

Note that

$$
\limsup _{n \rightarrow \infty} \int_{\Omega}-g_{n}(x) d x \leq \int_{\Omega} \limsup _{n \rightarrow \infty}\left(-g_{n}(x)\right) d x .
$$

$$
\begin{aligned}
\int_{\Omega} \limsup _{n \rightarrow \infty}\left(-g_{n}(x)\right) d x & =\int_{\Omega} \limsup _{n \rightarrow \infty}\left(A_{n}(x)-B_{n}(x)\right) d x \\
\int_{\Omega} \limsup _{n \rightarrow \infty} A_{n}(x) d x & \leq \int_{\Omega} \limsup _{\substack{\text { shu(x) } \\
t \rightarrow 0+}} \frac{F(x, y+t v(x))-F(x, y)}{t} d x \\
& =\int_{\Omega} F_{x}^{0}(x, u(x) ; v(x)) d x, \\
\int_{\Omega} \liminf _{n \rightarrow \infty} B_{n}(x) d x & =\int_{\Omega}\left(a_{0}+2 c_{1}|u(x)|^{\alpha(x)-1}\right)|v(x)| d x .
\end{aligned}
$$

Therefore

$$
\begin{aligned}
\int_{\Omega} \limsup _{n \rightarrow \infty}\left(-g_{n}(x)\right) d x \leq & \int_{\Omega} F_{x}^{0}(x, u(x) ; v(x)) d x \\
& -\int_{\Omega}\left(a_{0}+2 c_{1}|u(x)|^{\alpha(x)-1}\right)|v(x)| d x .
\end{aligned}
$$

For all $(x, t) \in \Omega \times \mathbb{R}$, define $f(x, t)=|v(x)| \cdot|t|^{\alpha(x)-1}$. Then there exists $c_{4}>0$ such that $|f(x, t)| \leq c_{4}\left(1+|v|^{p^{*}(x)}+|t|^{p^{*}(x)}\right)$. We know that the Nemytskiul operator

$$
N_{f}: L^{p^{*}(x)}(\Omega) \rightarrow L^{1}(\Omega): u \mapsto f(x, u)
$$

is continuous. By Theorem 1.1 in [8], $w_{n} \rightarrow u$ in $L^{p^{*}(x)}(\Omega)$, so 


$$
f\left(x, w_{n}+t_{n} v\right) \rightarrow f(x, u)
$$

in $L^{1}(\Omega)$. Thus

$$
\int_{\Omega}\left|w_{n}+t_{n} v\right|^{\alpha(x)-1}|v| d x \rightarrow \int_{\Omega}|u|^{\alpha(x)-1}|v| d x
$$

and $\int_{\Omega} B_{n}(x) d x \rightarrow \int_{\Omega}\left(a_{0}+2 c_{1}|u(x)|^{\alpha(x)-1}\right)|v(x)| d x$ as $n \rightarrow \infty$. Hence

$$
\limsup _{n \rightarrow \infty} \int_{\Omega}-g_{n}(x) d x=\psi^{0}(u ; v)-\int_{\Omega}\left(a_{0}+2 c_{1}|u(x)|^{\alpha(x)-1}\right)|v(x)| d x .
$$

Now the proof is complete.

THEOREM 3.3. Under condition (H1) or (H4), any critical point of $\varphi$ is a solution of (1.1).

Proof. It is easy to verify that $J \in C^{1}\left(W_{0}^{1, p(x)}(\Omega), \mathbb{R}\right)$. Combining this with Theorem 3.1, we find that $\varphi$ is locally Lipschitz. If $u$ is a critical point of $\varphi$, then $0 \in \partial \varphi(u)$. Thus for any $v \in W_{0}^{1, p(x)}(\Omega), \varphi^{0}(u ; v) \geq 0$. Noting that

$$
\begin{aligned}
\varphi^{0}(u ; v) & =\left\langle J^{\prime}(u), v\right\rangle+(-\psi)^{0}(u ; v)=\left\langle J^{\prime}(u), v\right\rangle+\psi^{0}(u ;-v) \\
& \leq \int_{\Omega}\left(|\nabla u|^{p(x)-2} \nabla u \nabla v+|u|^{p(x)-2} u v\right) d x+\int_{\Omega} F_{x}^{0}(x, u(x) ;-v(x)) d x,
\end{aligned}
$$

it is easy to get the result.

LEMma 3.1. Under conditions (H1), (H2), $\varphi$ satisfies the (P.S.) condition.

Proof. Take $\left\{u_{n}\right\} \subset W_{0}^{1, p(x)}(\Omega)$ such that $\varphi\left(u_{n}\right)$ is bounded and

$$
\lambda\left(u_{n}\right)=\min \left\{\left\|w^{*}\right\|_{W^{-1, p^{\prime}(x)}(\Omega)}: w^{*} \in \partial \varphi\left(u_{n}\right)\right\} \triangleq\left\|w_{n}^{*}\right\|_{W^{-1, p^{\prime}(x)}(\Omega)} \rightarrow 0
$$

as $n \rightarrow \infty$. Then

$$
\varphi^{0}\left(u_{n} ; v_{n}\right) \geq\left\langle w_{n}^{*}, u_{n}\right\rangle, \quad-\varphi^{0}\left(u_{n} ; v_{n}\right) \leq\left\|w_{n}^{*}\right\| \cdot\left|\nabla u_{n}\right|_{p(x)} .
$$

(i) $\left\{u_{n}\right\}$ is bounded in $W_{0}^{1, p(x)}(\Omega)$. In fact, as $\mu \gg p(x)$, we get

$$
\begin{aligned}
c_{5}+\left|\nabla u_{n}\right|_{p(x)} \geq & \varphi\left(u_{n}\right)-\frac{1}{\mu} \varphi^{0}\left(u_{n} ; u_{n}\right) \\
= & \varphi\left(u_{n}\right)-\left\langle J^{\prime}\left(u_{n}\right), \frac{u_{n}}{\mu}\right\rangle-\frac{1}{\mu} \psi^{0}\left(u_{n} ;-u_{n}\right) \\
\geq & \int_{\Omega}\left(\frac{1}{p(x)}-\frac{1}{\mu}\right)\left|\nabla u_{n}\right|^{p(x)} d x \\
& -\int_{\Omega}\left(F\left(x, u_{n}\right)+\frac{1}{\mu} F_{x}^{0}\left(x, u_{n}(x) ;-u_{n}(x)\right)\right) d x \\
\geq & \int_{\Omega}\left(\frac{1}{p(x)}-\frac{1}{\mu}\right)\left|\nabla u_{n}\right|^{p(x)} d x
\end{aligned}
$$


when $n$ is sufficiently large. It is easy to deduce that $\left\{u_{n}\right\}$ is bounded in $W_{0}^{1, p(x)}(\Omega)$.

(ii) $\left\{u_{n}\right\}$ has a convergent subsequence. In fact, as $W_{0}^{1, p(x)}(\Omega)$ is reflexive, passing to a subsequence, still denoted by $\left\{u_{n}\right\}$, we may assume that there exists $u \in W_{0}^{1, p(x)}(\Omega)$ such that $u_{n} \rightarrow u$ weakly in $W_{0}^{1, p(x)}(\Omega)$. Then $u_{n} \rightarrow u$ in $L^{\alpha(x)}(\Omega)$ and in $L^{p(x)}(\Omega)$. Noting that

$$
\begin{aligned}
\varphi^{0}\left(u_{n} ; u-u_{n}\right) & =\left\langle J^{\prime}\left(u_{n}\right), u-u_{n}\right\rangle+\psi^{0}\left(u_{n} ; u_{n}-u\right), \\
\varphi^{0}\left(u ; u_{n}-u\right) & =\left\langle J^{\prime}(u), u_{n}-u\right\rangle+\psi^{0}\left(u ; u-u_{n}\right),
\end{aligned}
$$

we get

$$
\begin{aligned}
& \left\langle J^{\prime}\left(u_{n}\right)-J^{\prime}(u), u_{n}-u\right\rangle \\
& =\psi^{0}\left(u_{n} ; u_{n}-u\right)+\psi^{0}\left(u ; u-u_{n}\right)-\varphi^{0}\left(u_{n} ; u-u_{n}\right)-\varphi^{0}\left(u ; u_{n}-u\right) .
\end{aligned}
$$

For all $w^{*} \in \partial \varphi(u), \varphi^{0}\left(u ; u_{n}-u\right) \geq\left\langle w^{*}, u_{n}-u\right\rangle$, so

$$
\liminf _{n \rightarrow \infty} \varphi^{0}\left(u ; u_{n}-u\right) \geq 0 \text {. }
$$

As $\varphi^{0}\left(u_{n} ; u-u_{n}\right) \geq\left\langle w_{n}^{*}, u-u_{n}\right\rangle \geq-c_{5}\left\|w_{n}^{*}\right\|$, we have

$$
\liminf _{n \rightarrow \infty} \varphi^{0}\left(u_{n} ; u-u_{n}\right) \geq 0 .
$$

By Theorem 3.2, we get

$$
\begin{aligned}
\psi^{0}\left(u_{n} ; u_{n}-u\right)+\psi^{0}\left(u ; u-u_{n}\right) \\
\leq \int_{\Omega} F_{x}^{0}\left(x, u_{n}(x) ; u_{n}(x)-u(x)\right) d x+\int_{\Omega} F_{x}^{0}\left(x, u(x) ; u(x)-u_{n}(x)\right) d x \\
\leq \int_{\Omega} \max \left\{\xi \cdot\left(u_{n}(x)-u(x)\right): \xi \in \partial F\left(x, u_{n}(x)\right)\right\} d x \\
\quad \quad+\int_{\Omega} \max \left\{\xi \cdot\left(u(x)-u_{n}(x)\right): \xi \in \partial F(x, u(x))\right\} d x \\
\leq \int_{\Omega} c_{6}\left(1+\left|u_{n}\right|^{\alpha(x)-1}+|u|^{\alpha(x)-1}\right)\left|u_{n}-u\right| d x \\
\leq\left. c_{7}|1+| u_{n}\right|^{\alpha(x)-1}+\left.|u|^{\alpha(x)-1}\right|_{\alpha^{\prime}(x)} \cdot\left|u_{n}-u\right|_{\alpha(x)} \leq c_{8}\left|u_{n}-u\right|_{\alpha(x)} \rightarrow 0 .
\end{aligned}
$$

Thus $\lim \sup _{n \rightarrow \infty}\left\langle J^{\prime}\left(u_{n}\right)-J^{\prime}(u), u_{n}-u\right\rangle \leq 0$. Similar to Theorem 3.1 in [1], we conclude that $u_{n} \rightarrow u$ in $W_{0}^{1, p(x)}(\Omega)$.

Lemma 3.2. We have $\varphi(0)=0$. Under conditions (H1), (H3), there exist $r_{1}, s_{1}>0$ such that $\varphi(u)>0$ for $0<|\nabla u|_{p(x)} \leq r_{1}$ and $\varphi(u)>s_{1}$ for $|\nabla u|_{p(x)}=r_{1}$.

Proof. It is easy to show that $\varphi(0)=0$. By condition (H3), for all $\varepsilon>0$, there exists $\delta>0$ such that

$$
\max \{|\xi|: \xi \in \partial F(x, t)\} \leq \varepsilon|t|^{p(x)-1}
$$


for all $|t|<\delta$ and $x \in \Omega$. By Lebourg's mean value theorem, there exist $\theta \in(0,1)$ and $\xi_{\theta} \in \partial F(x, \theta t)$ such that $F(x, t)=\xi_{\theta} t$. Combining this with condition (H1), we see that for all $\varepsilon>0$, there exists $c_{9}>0$ such that

$$
|F(x, t)| \leq \varepsilon|t|^{p(x)}+c_{9}|t|^{\alpha(x)}
$$

for all $(x, t) \in \Omega \times \mathbb{R}$. For $\varepsilon<1 / p_{+}$, we get

$$
\begin{aligned}
\varphi(u) & \geq \int_{\Omega}\left(\frac{|\nabla u|^{p(x)}+|u|^{p(x)}}{p_{+}}-\varepsilon|u|^{p(x)}-c_{9}|u|^{\alpha(x)}\right) d x \\
& \geq \int_{\Omega}\left(\frac{|\nabla u|^{p(x)}}{p_{+}}-c_{9}|u|^{\alpha(x)}\right) d x .
\end{aligned}
$$

As $p(x) \ll \alpha(x)$, similar to Theorem 3.1 in [1], we get the result.

LEMMA 3.3. Under condition (H2), there exists $e \in W_{0}^{1, p(x)}(\Omega)$ such that $\varphi(e)<0$.

Proof. Fix $x_{0} \in \Omega_{0}$ and $0<R<1 / 2$ such that $B_{2 R}\left(x_{0}\right) \subset \Omega_{0}$. Let $\phi \in C_{0}^{\infty}\left(B_{2 R}\left(x_{0}\right)\right), 0 \leq \phi(x) \leq 1,|\nabla \phi(x)| \leq 1 / R$, and suppose $\phi(x) \equiv 1$ for $x \in B_{R}\left(x_{0}\right)$. For $t>1$, it is easy to get

$$
\begin{aligned}
\varphi(t \phi) & =\int_{B_{2 R}\left(x_{0}\right)}\left(\frac{|t \nabla \phi|^{p(x)}+|t \phi|^{p(x)}}{p(x)}-F(x, t \phi)\right) d x \\
& \leq \int_{B_{2 R}\left(x_{0}\right)}\left(c_{10} t^{p_{+}}-a_{2}|t \phi|^{\mu}+a_{3}\right) d x .
\end{aligned}
$$

As $\mu \gg p(x)$, we get $\varphi(t \phi)<0$, when $t$ is sufficiently large.

Proof of Theorem 1.1. By Lemmata 3.1-3.3 and Proposition 2.1, we easily get the result.

LEMma 3.4. Under condition (H4), the functional $\varphi$ is bounded from below.

Proof. By Lebourg's mean value theorem, there exist $\theta \in(0,1)$ and $\xi_{\theta} \in \partial F(x, \theta t)$ such that $F(x, t)=\xi_{\theta} t$. Hence

$$
|F(x, t)| \leq b_{0}|t|+b_{1}|t|^{\beta(x)}
$$

for all $(x, t) \in \Omega \times \mathbb{R}$. Thus

$$
\varphi(u) \geq \int_{\Omega}\left(\frac{|\nabla u|^{p(x)}+|u|^{p(x)}}{p(x)}-b_{0}|u|-b_{1}|u|^{\beta(x)}\right) d x
$$

for all $u \in W_{0}^{1, p(x)}(\Omega)$. As $\beta(x) \ll p(x)$, similar to Theorem 3.2 in [1], there 
exists $c_{11}>0$ such that

$$
\varphi(u) \geq \int_{\Omega} \frac{|\nabla u|^{p(x)}}{p_{+}} d x-c_{11}
$$

for all $u \in W_{0}^{1, p(x)}(\Omega)$. Thus we get the result.

Lemma 3.5. Under condition (H5), there exists $e \in W_{0}^{1, p(x)}(\Omega)$ such that $\varphi(e)<0$.

Proof. Fix $x_{0} \in \Omega_{0}$ and $R>0$ such that $B_{2 R}\left(x_{0}\right) \subset \Omega_{0}$ and

$$
\beta_{1}=\sup _{x \in B_{2 R}\left(x_{0}\right)} \beta(x)<p_{1}=\inf _{x \in B_{2 R}\left(x_{0}\right)} p(x) .
$$

Let $\phi \in C_{0}^{\infty}\left(B_{2 R}\left(x_{0}\right)\right), 0 \leq \phi(x) \leq 1,|\nabla \phi(x)| \leq 1 / R$, and suppose $\phi(x) \equiv 1$ for $x \in B_{R}\left(x_{0}\right)$. For $0<t<\min \{1, \delta\}$, by condition (H5),

$$
\begin{aligned}
\varphi(t \phi) & =\int_{B_{2 R}\left(x_{0}\right)}\left(\frac{|t \nabla \phi|^{p(x)}}{p(x)}+\frac{|t \phi|^{p(x)}}{p(x)}-F(x, t \phi)\right) d x \\
& \leq \int_{B_{2 R}\left(x_{0}\right)}\left(c_{12} t^{p(x)}-b_{2}(t \phi)^{\beta(x)}\right) d x \\
& \leq t^{\beta_{1}} \int_{B_{2 R}\left(x_{0}\right)}\left(c_{12} t^{p_{1}-\beta_{1}}-b_{2} \phi^{\beta(x)}\right) d x .
\end{aligned}
$$

As $\phi(x) \equiv 1$ for $x \in B_{R}\left(x_{0}\right)$, we have $\int_{B_{2}\left(x_{0}\right)} \phi^{\beta(x)} d x>0$. When $t$ is sufficiently small, we get $\varphi(t \phi)<0$.

Proof of Theorem 1.2. Similar to Lemma 3.1, it is easy to verify that the functional $\varphi$ satisfies the (P.S.) condition. Combining this with Lemmata 3.4, 3.5 and Proposition 2.2, we know that

$$
c=\inf _{u \in W_{0}^{1, p(x)}(\Omega)} \varphi(u)<0
$$

is a critical value of $\varphi$. Now the proof is complete.

4. The case of exterior domain. Throughout this section, we assume that $\Omega \subset \mathbb{R}^{N}$ is an exterior domain and denote by $d_{i}$ various positive constants.

TheOREM 4.1. Under condition ( $\mathrm{H} 6)$ or $(\mathrm{H} 7), \psi$ is well defined and is a locally Lipschitz functional on $W_{0}^{1, p(x)}(\Omega)$.

Proof. We only consider the case where the functional $F$ satisfies (H6). 
(i) $\psi$ is well defined. By Lebourg's mean value theorem, for all $t_{1}, t_{2} \in \mathbb{R}$, there exist $\theta \in(0,1)$ and $\xi_{\theta} \in \partial F\left(x, \theta t_{1}+(1-\theta) t_{2}\right)$ such that

$$
F\left(x, t_{1}\right)-F\left(x, t_{2}\right)=\xi_{\theta}\left(t_{1}-t_{2}\right)
$$

for all $x \in \Omega$. By condition (H6), we get

$$
\begin{aligned}
\left|F\left(x, t_{1}\right)-F\left(x, t_{2}\right)\right| & \leq g(x)\left|\theta t_{1}+(1-\theta) t_{2}\right|^{\alpha(x)-1} \cdot\left|t_{1}-t_{2}\right| \\
& \leq d_{1} g(x)\left(\left|t_{1}\right|^{\alpha(x)-1}+\left|t_{2}\right|^{\alpha(x)-1}\right)\left|t_{1}-t_{2}\right| .
\end{aligned}
$$

By the Young inequality, we also get

$$
|\psi(u)| \leq \int_{\Omega}|F(x, u)| d x \leq \int_{\Omega} d_{1} g(x)|u|^{\alpha(x)} d x \leq \int_{\Omega} d_{2}\left(g(x)^{q_{1}(x)}+|u|^{p^{*}(x)}\right) d x .
$$

Thus by Theorem 1.1 in [8], we have $|\psi(u)|<\infty$ for all $u \in W_{0}^{1, p(x)}(\Omega)$.

(ii) $\psi$ is locally Lipschitz on $W_{0}^{1, p(x)}(\Omega)$. In fact, for all $u_{1}, u_{2} \in W_{0}^{1, p(x)}(\Omega)$,

$$
\begin{aligned}
\left|\psi\left(u_{1}\right)-\psi\left(u_{2}\right)\right| & \leq \int_{\Omega}\left|F\left(x, u_{1}\right)-F\left(x, u_{2}\right)\right| d x \\
& \leq \int_{\Omega} d_{1} g(x)\left(\left|u_{1}\right|^{\alpha(x)-1}+\left|u_{2}\right|^{\alpha(x)-1}\right)\left|u_{1}-u_{2}\right| d x \\
& \leq\left. d_{3}|g(x)| u_{1}\right|^{\alpha(x)-1}+\left.g(x)\left|u_{2}\right|^{\alpha(x)-1}\right|_{\left(p^{*}(x)\right)^{\prime}} \cdot\left|u_{1}-u_{2}\right|_{p^{*}(x)} \\
& \leq\left. d_{4}|g(x)| u_{1}\right|^{\alpha(x)-1}+\left.g(x)\left|u_{2}\right|^{\alpha(x)-1}\right|_{\left(p^{*}(x)\right)^{\prime}} \cdot\left\|u_{1}-u_{2}\right\|_{1, p(x)} .
\end{aligned}
$$

By the Young inequality, we get

$$
\int_{\Omega}\left(g(x)\left|u_{1}\right|^{\alpha(x)-1}\right)^{\left(p^{*}(x)\right)^{\prime}} d x \leq \int_{\Omega} d_{5}\left(g(x)^{q_{1}(x)}+\left|u_{1}\right|^{p^{*}(x)}\right) d x .
$$

As $g \in L^{q_{1}(x)}(\Omega)$ and the imbedding $W_{0}^{1, p(x)}(\Omega) \hookrightarrow L^{p^{*}(x)}(\Omega)$ is continuous, we can easily get the result.

THEOREM 4.2. Under condition $(\mathrm{H} 6)$ or $(\mathrm{H} 7)$, for all $u, v \in W_{0}^{1, p(x)}(\Omega)$, we have

$$
\psi^{0}(u ; v) \leq \int_{\Omega} F_{x}^{0}(x, u(x) ; v(x)) d x .
$$

Proof. (i) Similar to Theorem 3.1, we prove that $F_{x}^{0}(x, u(x) ; v(x))$ is measurable. Here we only consider the case where the functional $F$ satisfies (H6).

Noting that $F_{x}^{0}(x, u(x) ; v(x))=\max \{\xi \cdot v(x): \xi \in \partial F(x, u(x))\} \triangleq$ $\xi_{x} \cdot v(x)$, we get

$$
\begin{aligned}
\left|F_{x}^{0}(x, u(x) ; v(x))\right| & =\left|\xi_{x} \cdot v(x)\right| \leq g(x)|u(x)|^{\alpha(x)-1}|v(x)| \\
& \leq d_{6}\left(g(x)^{q_{1}(x)}+|u|^{p^{*}(x)}+|v|^{p^{*}(x)}\right) .
\end{aligned}
$$

Hence $F_{x}^{0}(x, u(x) ; v(x)) \in L^{1}(\Omega)$. 
(ii) $\psi^{0}(u ; v) \leq \int_{\Omega} F_{x}^{0}(x, u(x) ; v(x)) d x$. By the definition of $\psi^{0}(u ; v)$, there exist $t_{n} \rightarrow 0+$ and $w_{n} \rightarrow u$ in $W_{0}^{1, p(x)}(\Omega)$ such that

$$
\varphi^{0}(u ; v)=\lim _{\substack{w_{n} \rightarrow u \\ t_{n} \rightarrow 0+}} \frac{\varphi\left(w_{n}+t_{n} v\right)-\varphi\left(w_{n}\right)}{t_{n}} .
$$

Passing to a subsequence, still denoted by $\left\{w_{n}\right\}$, we may assume that $w_{n}(x) \rightarrow u(x)$ a.e. in $\Omega$ as $n \rightarrow \infty$. Set

$$
\begin{aligned}
A_{n}(x) & =\frac{F\left(x, w_{n}(x)+t_{n} v(x)\right)-F\left(x, w_{n}(x)\right)}{t_{n}}, \\
B_{n}(x) & =d_{1} g(x)\left(\left|w_{n}(x)+t_{n} v(x)\right|^{\alpha(x)-1}+\left|w_{n}(x)\right|^{\alpha(x)-1}\right)|v(x)|, \\
g_{n}(x) & =-A_{n}(x)+B_{n}(x) .
\end{aligned}
$$

Then similar to the proof of Theorem 3.2, we get the result.

Lemma 4.1. Under condition (H6), $\varphi$ satisfies the (P.S.) condition.

Proof. Take $\left\{u_{n}\right\} \subset W_{0}^{1, p(x)}(\Omega)$ such that $\varphi\left(u_{n}\right)$ is bounded and

$$
\lambda\left(u_{n}\right)=\min \left\{\left\|w^{*}\right\|_{W^{-1, p^{\prime}(x)}(\Omega)}: w^{*} \in \partial \varphi\left(u_{n}\right)\right\} \triangleq\left\|w_{n}^{*}\right\|_{W^{-1, p^{\prime}(x)}(\Omega)} \rightarrow 0
$$

as $n \rightarrow \infty$. Then similar to Lemma 3.1, we see that $\left\{u_{n}\right\}$ is bounded in $W_{0}^{1, p(x)}(\Omega)$.

As $W_{0}^{1, p(x)}(\Omega)$ is reflexive, passing to a subsequence, still denoted by $\left\{u_{n}\right\}$, we may assume that there exists $u \in W_{0}^{1, p(x)}(\Omega)$ such that $u_{n} \rightarrow u$ weakly in $W_{0}^{1, p(x)}(\Omega)$. Then $u_{n} \rightarrow u$ in $L^{\alpha(x)}(\Omega)$ and in $L^{p(x)}(\Omega)$. Noting that

$$
\begin{aligned}
\varphi^{0}\left(u_{n} ; u-u_{n}\right) & =\left\langle J^{\prime}\left(u_{n}\right), u-u_{n}\right\rangle+\psi^{0}\left(u_{n} ; u_{n}-u\right), \\
\varphi^{0}\left(u ; u_{n}-u\right) & =\left\langle J^{\prime}(u), u_{n}-u\right\rangle+\psi^{0}\left(u ; u-u_{n}\right),
\end{aligned}
$$

we get

$$
\begin{aligned}
& \left\langle J^{\prime}\left(u_{n}\right)-J^{\prime}(u), u_{n}-u\right\rangle \\
& \quad=\psi^{0}\left(u_{n} ; u_{n}-u\right)+\psi^{0}\left(u ; u-u_{n}\right)-\varphi^{0}\left(u_{n} ; u-u_{n}\right)-\varphi^{0}\left(u ; u_{n}-u\right) .
\end{aligned}
$$

For all $w^{*} \in \partial \varphi(u), \varphi^{0}\left(u ; u_{n}-u\right) \geq\left\langle w^{*}, u_{n}-u\right\rangle$, so

$$
\liminf _{n \rightarrow \infty} \varphi^{0}\left(u ; u_{n}-u\right) \geq 0 .
$$

As $\varphi^{0}\left(u_{n} ; u-u_{n}\right) \geq\left\langle w_{n}^{*}, u-u_{n}\right\rangle \geq-d_{7}\left\|w_{n}^{*}\right\|$, we have

$$
\liminf _{n \rightarrow \infty} \varphi^{0}\left(u_{n} ; u-u_{n}\right) \geq 0 .
$$


Moreover,

$$
\begin{aligned}
& \psi^{0}\left(u_{n} ; u_{n}-u\right)+\psi^{0}\left(u ; u-u_{n}\right) \\
& \leq \int_{\Omega} F_{x}^{0}\left(x, u_{n}(x) ; u_{n}(x)-u(x)\right) d x+\int_{\Omega} F_{x}^{0}\left(x, u(x) ; u(x)-u_{n}(x)\right) d x \\
& \leq \int_{\Omega} \max \left\{\xi \cdot\left(u_{n}(x)-u(x)\right): \xi \in \partial F\left(x, u_{n}(x)\right)\right\} d x \\
& \quad+\int_{\Omega} \max \left\{\xi \cdot\left(u(x)-u_{n}(x)\right): \xi \in \partial F(x, u(x))\right\} d x \\
& \leq \int_{\Omega} g(x)\left(\left|u_{n}\right|^{\alpha(x)-1}+|u|^{\alpha(x)-1}\right)\left|u_{n}-u\right| d x .
\end{aligned}
$$

Similar to Theorem 4.3 in [11], we get

$$
\int_{\Omega} g(x)\left(\left|u_{n}\right|^{\alpha(x)-1}+|u|^{\alpha(x)-1}\right)\left|u_{n}-u\right| d x \rightarrow 0
$$

as $n \rightarrow \infty$. Then $\lim \sup _{n \rightarrow \infty}\left\langle J^{\prime}\left(u_{n}\right)-J^{\prime}(u), u_{n}-u\right\rangle \leq 0$, and similar to Theorem 3.1 in [10], it is easy to get $u_{n} \rightarrow u$ in $W_{0}^{1, p(x)}(\Omega)$.

Proof of Theorem 1.3. Firstly, we assert that there exist $r_{2}, s_{2}>0$ such that $\varphi(u)>0$ for $0<|\nabla u|_{p(x)} \leq r_{2}$ and $\varphi(u)>s_{2}$ for $|\nabla u|_{p(x)}=r_{2}$.

In fact, by Lebourg's mean value theorem, there exist $\theta \in(0,1)$ and $\xi_{\theta} \in \partial F(x, \theta t)$ such that $F(x, t)=\xi_{\theta} t$ for all $(x, t) \in \Omega \times \mathbb{R}$. Combining this with condition (H6), we obtain

$$
|F(x, t)| \leq g(x)|t|^{\alpha(x)} \leq d_{8}|t|^{\alpha(x)}
$$

for all $(x, t) \in \Omega \times \mathbb{R}$. Thus

$$
\begin{aligned}
\varphi(u) & \geq \int_{\Omega}\left(\frac{|\nabla u|^{p(x)}+|u|^{p(x)}}{p_{+}}-d_{8}|u|^{\alpha(x)}\right) d x \\
& =\int_{\Omega} \frac{|\nabla u|^{p(x)}+|u|^{p(x)}}{2 p_{+}} d x+\int_{\Omega}\left(\frac{|\nabla u|^{p(x)}+|u|^{p(x)}}{2 p_{+}}-d_{8}|u|^{\alpha(x)}\right) d x,
\end{aligned}
$$

and similar to Theorem 3.1 in [10], we easily get the above assertion. Then by Lemmata 3.3, 4.1 and Proposition 2.1, we complete the proof.

Proof of Theorem 1.4. Firstly, we need to verify that the functional $\varphi$ is coercive. By Lebourg's mean value theorem, there exist $\theta \in(0,1)$ and $\xi_{\theta} \in \partial F(x, \theta t)$ such that $F(x, t)=\xi_{\theta} t$. Hence for all $(x, t) \in \Omega \times \mathbb{R}$, we get

$$
|F(x, t)| \leq h(x)|t|^{\beta(x)} .
$$

Thus

$$
\varphi(u) \geq \int_{\Omega}\left(\frac{|\nabla u|^{p(x)}+|u|^{p(x)}}{p(x)}-h(x)|u|^{\beta(x)}\right) d x
$$


for all $u \in W_{0}^{1, p(x)}(\Omega)$. As $\beta(x) \ll p(x)$, similar to Lemma 4.3 in [11], there exists $d_{9}>0$ such that

$$
\varphi(u) \geq \int_{\Omega} \frac{|\nabla u|^{p(x)}+|u|^{p(x)}}{2 p_{+}} d x-d_{9}
$$

for all $u \in W_{0}^{1, p(x)}(\Omega)$. Then similar to the argument for Theorem 1.2, the proof is complete.

5. Some examples. In this section, we give some concrete examples of functionals $F$ satisfying the assumptions of Theorems 1.1-1.4.

EXAMPLE 5.1. Let $\Omega \subset \mathbb{R}^{N}$ be a bounded domain. Define $F: \bar{\Omega} \times \mathbb{R} \rightarrow \mathbb{R}$ by

$$
F(x, t)=\frac{|t|^{\alpha(x)}}{\alpha(x)},
$$

where $\alpha \in C(\bar{\Omega})$ with $p_{+}<\alpha_{-}$. We can check that $-F_{x}^{0}(x, t ;-t)=|t|^{\alpha(x)}$ and $\partial F(x, t)=\left\{|t|^{\alpha(x)-2} t\right\}$. If we let $\mu=\alpha_{-}$, it is easy to verify that $F$ satisfies the assumptions in Theorem 1.1.

EXAMPLE 5.2. Let $\Omega \subset \mathbb{R}^{N}$ be a bounded domain. Let $F: \bar{\Omega} \times \mathbb{R} \rightarrow \mathbb{R}$ be defined by

$$
F(x, t)=\frac{|t|^{\beta_{-}}}{\beta_{-}}+\frac{|t|^{\beta(x)}}{\beta(x)},
$$

where $\beta \in \mathbf{P}(\Omega)$ with $1<\beta_{-} \leq \beta(x) \ll p(x)$. Then we can verify that

$$
\partial F(x, t)=\left\{|t|^{\beta_{-}-2} t+|t|^{\beta(x)-2} t\right\}
$$

and $F$ satisfies the assumptions in Theorem 1.2.

ExAmple 5.3. Let $\Omega=\mathbb{R}^{N} \backslash B(0,1)$, where $B(0,1)$ is the closed unit ball in $\mathbb{R}^{N}$. Define $F: \bar{\Omega} \times \mathbb{R} \rightarrow \mathbb{R}$ by

$$
F(x, t)=g(x) \frac{|t|^{\alpha(x)}}{\alpha(x)},
$$

where $\alpha \in \mathbf{P}(\Omega)$ with $p(x) \ll \alpha(x) \ll p^{*}(x), g(x)=|x|^{-N}$. Then it is easy to see that $g \in L^{\infty}(\Omega) \cap L^{q_{1}(x)}(\Omega)$ with $q_{1}(x)=p^{*}(x) /\left(p^{*}(x)-\alpha(x)\right)$. Thus we can apply Theorem 1.3. If we choose $\alpha \in \mathbf{P}(\Omega)$ with $1<\alpha_{-} \leq \alpha(x) \ll p(x)$, then $F$ satisfies the assumptions of Theorem 1.4.

Based on Theorems 1.1-1.4, we can solve a larger class of hemivariational inequalities which have $p(x)$-growth conditions. In particular, Theorems 1.1-1.4 are also applicable when the function $p(x)$ is a constant. 
Remark. In particular, if $F: \bar{\Omega} \times \mathbb{R} \rightarrow \mathbb{R}$ is defined by

$$
F(x, t)=\int_{0}^{t} f(x, s) d s
$$

where $\Omega \subset \mathbb{R}^{N}$ is a domain and $f \in C(\bar{\Omega} \times \mathbb{R}, \mathbb{R})$, then the inequality (1.1) takes the form

$$
\int_{\Omega}\left(|\nabla u|^{p(x)-2} \nabla u \nabla v+|u|^{p(x)-2} u v\right) d x+\int_{\Omega} f(x, u) v d x=0
$$

for all $v \in W_{0}^{1, p(x)}(\Omega)$, i.e. $u \in W_{0}^{1, p(x)}(\Omega)$ is a weak solution of

$$
\left\{\begin{array}{l}
-\operatorname{div}\left(|\nabla w|^{p(x)-2} \nabla w\right)+|w|^{p(x)-2} w=f(x, w), \\
w \in W_{0}^{1, p(x)}(\Omega) .
\end{array}\right.
$$

The existence of solutions for the above equation has been studied recently: we refer to $[1,7,10,11,18]$.

Acknowledgements. This research was supported by Science Research Foundation in Harbin Institute of Technology (HITC200702) and The Natural Science Foundation of Heilongjiang Province (A2007-04).

\section{References}

[1] J. Chabrowski and Y. Q. Fu, Existence of solutions for $p(x)$-Laplacian problems on a bounded domain, J. Math. Anal. Appl. 306 (2005), 604-618; Erratum, ibid. 323 (2006), 1483.

[2] K. Chang, Variational methods for non-differential functionals and their applications to partial differential equations, ibid. 80 (1981), 102-129.

[3] F. H. Clarke, A new approach to Lagrange multipliers, Math. Oper. Res. 1 (1976), $165-173$.

[4] -, Nonsmooth Analysis and Optimization, Wiley, New York, 1983.

[5] D. Edmunds, J. Lang and A. Nekvinda, On $L^{p(x)}$ norms, Proc. Roy. Soc. London Ser. A 455 (1999), 219-225.

[6] D. Edmunds and J. Rákosník, Sobolev embedding with variable exponent, Studia Math. 143 (2000), 267-293.

[7] X. L. Fan and X. Y. Han, Existence and multiplicity of solutions for $p(x)$-Laplacian equations in $\mathbb{R}^{N}$, Nonlinear Anal. 59 (2004), 173-188.

[8] X. L. Fan, J. S. Shen and D. Zhao, Sobolev embedding theorems for spaces $W^{k, p(x)}(\Omega)$, J. Math. Anal. Appl. 262 (2001), 749-760.

[9] X. L. Fan and Q. H. Zhang, Existence of solutions for $p(x)$-Laplacian Dirichlet problem, Nonlinear Anal. 52 (2003), 1843-1852.

[10] Y. Q. Fu, Existence of solutions for $p(x)$-Laplacian problem on an unbounded domain, Topol. Methods Nonlinear Anal. 30 (2007), 235-249.

[11] Y. Q. Fu and X. Zhang, A multiplicity result for $p(x)$-Laplacian problem in $\mathbb{R}^{N}$, Nonlinear Anal., in press.

[12] O. Kováčik and J. Rákosník, On spaces $L^{p(x)}$ and $W^{k, p(x)}$, Czechoslovak Math. J. 41 (1991), 592-618. 
[13] A. Kristály, Infinitely many radial and non-radial solutions for a class of hemivariational inequalities, Rocky Mountain J. Math. 35 (2005), 1174-1190.

[14] M. Mihăilescu and V. Rădulescu, A multiplicity result for a nonlinear degenerate problem arising in the theory of electrorheological fluids, Proc. Roy. Soc. London Ser. A 462 (2006), 2625-2641.

[15] Z. Naniewicz and P. D. Panagiotopoulos, Mathematical Theory of Hemivariational Inequalities and Applications, Dekker, New York, 1995.

[16] P. D. Panagiotopoulos, Hemivariational Inequalities: Applications to Mechanics and Engineering, Springer, New York, 1993.

[17] M. Růžička, Electro-Rheological Fluids: Modeling and Mathematical Theory, Springer, 2000.

[18] Q. H. Zhang, Existence of radial solutions for $p(x)$-Laplacian equations in $\mathbb{R}^{N}$, J. Math. Anal. Appl. 315 (2006), 506-516.

Department of Mathematics

Harbin Institute of Technology

Harbin 150001, China

E-mail: piecesummer1984@163.com

Received 18.9.2008

and in final form 6.11.2008 